\title{
Research Article \\ Effect of Experimentally Induced Hepatic and Renal Failure on the Pharmacokinetics of Topiramate in Rats
}

\author{
Kamal M. Matar ${ }^{1}$ and Yasin I. Tayem ${ }^{2}$ \\ ${ }^{1}$ Department of Pharmacology and Therapeutics, Faculty of Pharmacy, Kuwait University, P.O. Box 24923, Safat 13110, Kuwait \\ ${ }^{2}$ Department of Physiology and Pharmacology, School of Medicine, Al-Quds University, Abu Dees, West Bank, Jerusalem, Palestine \\ Correspondence should be addressed to Kamal M. Matar; kamal@hsc.edu.kw
}

Received 23 August 2013; Revised 7 April 2014; Accepted 24 May 2014; Published 9 June 2014

Academic Editor: Manjari Tripathi

Copyright (c) 2014 K. M. Matar and Y. I. Tayem. This is an open access article distributed under the Creative Commons Attribution License, which permits unrestricted use, distribution, and reproduction in any medium, provided the original work is properly cited.

\begin{abstract}
We aimed to investigate the effect of induced hepatic and renal failure on the pharmacokinetics of topiramate (TPM) in rats. Twentyfour Sprague-Dawley rats were used in this study. Renal or hepatic failure was induced by a single i.p. dose of $7.5 \mathrm{mg} / \mathrm{kg}$ cisplatin $(n=$ 8 ) or $0.5 \mathrm{~mL} / \mathrm{kg}$ carbon tetrachloride $\left(\mathrm{CCl}_{4}\right)(n=8)$, respectively. Three days after cisplatin dose or $24 \mathrm{~h}$ after $\mathrm{CCl}_{4}$ dose, the rats were administered a single oral dose of $20 \mathrm{mg} / \mathrm{kg}$ TPM. The plasma samples were quantified by LC-MS/MS method. Compared to control, plasma concentration-time profile in $\mathrm{CCl}_{4}$-treated and, to a lesser extent, in cisplatin-treated rats decreased more slowly particularly in the elimination phase. TPM oral clearance $(\mathrm{CL} / \mathrm{F})$ in $\mathrm{CCl}_{4}$-treated group was significantly lower than that in control $(P<0.001)$, whereas $\mathrm{AUC}_{0-\infty}, \mathrm{T} 1 / 2$, and $\mathrm{Vd} / \mathrm{F}$ were significantly higher in $\mathrm{CCl}_{4}$-treated rats compared to the control $(P<0.01)$. The $\mathrm{CL} / \mathrm{F}$ was not significantly different between cisplatin-treated rats and control $(P>0.05)$. However, in cisplatin-treated rats, the T1/2 and Vd/F were significantly higher than that in the control group $(P<0.01)$. Both conditions failed to cause a significant effect on $C_{\max }$ or $T_{\max }$. The present findings suggest that induced hepatic or renal failure could modify the pharmacokinetic profile of TPM in the rat.
\end{abstract}

\section{Introduction}

Topiramate (TPM) is a structurally novel broad spectrum anticonvulsant that is effective in the treatment of several types of epilepsy including partial onset [1] and primary generalized seizures [2]. TPM, which was originally designed as an oral hypoglycemic agent [3], was found to be an effective and tolerable antiepileptic drug, both as monotherapy and as add-on, in children and adults [4].

The pharmacokinetic profile of TPM in humans has been extensively characterized. Following oral administration, TPM is rapidly absorbed with high bioavailability $(\sim 80 \%)$ and low binding to plasma proteins [5]. Peak plasma concentration is reached within $1-4 \mathrm{~h}$ after administration [5]. In the dose range of 100 to $1200 \mathrm{mg}$, the mean apparent volume of distribution (Vd) is between 0.6 and $1.0 \mathrm{~L} / \mathrm{kg}$ [5]. At steady-state concentration, renal clearance of this drug is $1.02 \mathrm{~L} / \mathrm{h}[6]$ and its elimination half-life (T1/2) varies from 20 to $30 \mathrm{~h}$ [5]. In all species, TPM is predominantly excreted unchanged in urine [6].
Liver metabolic pathways of TPM in humans and animals are similar and involve hydroxylation or hydrolysis of isopropylidene groups [7]. Out of these hepatic metabolizing systems, cytochrome P450 (CYP) enzymes play a major role in the biotransformation and inactivation of this agent [5]. In general, alterations in the activity of CYP, as a result of liver diseases, decrease drug metabolism thus requiring dosage adjustment and therapeutic drug monitoring (TDM) [8]. The mechanisms by which liver diseases modify drug pharmacokinetics occur alone or in combination and encompass, in addition to altering CYP activity, changes in hepatic blood flow and binding of drugs to plasma proteins [8]. The latter influences the processes of drug distribution and elimination. The ultimate effect of these factors leads to a decrease in plasma clearance and an increase in the extent of absorption of drugs by decreasing their first pass effect following oral administration. In addition, patients with advanced liver disease often suffer from deterioration in renal function and dose adjustment becomes necessary even for drugs which are predominantly excreted unchanged in urine [9]. 
Since kidney is the major route of drug excretion from the body, any deterioration in renal function, even if it is mild, dramatically influences the pharmacokinetic characteristics of a drug and its metabolites. In fact, this is one of the most important factors which influence patient's response to an administered drug. Various pharmacokinetic parameters can be altered in response to renal impairment including absorption [10], bioavailability [11], Vd [12], and metabolism [13]. These alterations often require dose adjustment to avoid drug accumulation and serious toxicity. For these reasons, pharmacokinetic studies in renal impairment patients are an integral part of new drug development programs [14]. A key objective of these studies is to determine if disruption in renal function can alter pharmacokinetics of a drug sufficiently to require dosage adjustment.

To our knowledge, the effect of renal and hepatic failure on the pharmacokinetics of TPM has not been fully elucidated yet. For this reason, the present investigation was carried out to provide insight into the influence of compromised liver and kidney function on the pharmacokinetic profile of TPM in rats.

\section{Materials and Methods}

2.1. Chemicals and Reagents. The commercial formulation of TPM, Topamax (Janssen-Cilag, Schaffhausen, Switzerland) containing $100 \mathrm{mg}$ TPM, was locally purchased from a drug store. $\mathrm{CCl}_{4}$ and carboxymethylcellulose (CMC) were purchased from Sigma-Aldrich Company (Poole, UK). Cisplatin, the commercial formulation of cisplatin (Ebewe Pharma, $\mathrm{GmbH}$, Unterach, Austria) containing $50 \mathrm{mg} / 100 \mathrm{~mL}$ cisplatin injection, was locally purchased. TPM- $\mathrm{d}_{12}$ (internal standard "IS" for TPM analysis) was purchased from BDG Synthesis (Wellington, New Zealand). Water was purified using a Milli-Q water device (Millipore, Bedford, MA, USA). All other chemicals and reagents were of analytical grade and solvents were of HPLC grade. TPM was freshly prepared from tablet formulation suspended in $0.5 \% \mathrm{CMC} \mathrm{CCl}_{4}$ was dissolved in olive oil $(1: 1 \mathrm{v} / \mathrm{v})$ and cisplatin was used directly from a bottle for injection $(50 \mathrm{mg} / 100 \mathrm{~mL})$ without further dilution. Serum creatinine $(\mathrm{Scr})$ and aspartate aminotransferase (AST) were measured spectrophotometrically using diagnostic kits purchased from Human GmbH (Wiesbaden, Germany).

2.2. Ethical Approval. Ethical approval for conducting this study was provided by the local Experimental Animal Resource Center Ethics Committee, Health Sciences Center, Kuwait University, and it was in compliance with the Helsinki Declaration for Ethical Principles of Medical Research.

2.3. Animals and Treatment. Male Sprague-Dawley rats $(n=$ 24), weighing between 180 and $230 \mathrm{~g}$, were used in this study and were housed in a pathogen-free facility. The rats were acclimatized at least for one week before experiments in an animal facility in which the appropriate temperature, humidity, and light cycle (6:00 A.M.-6:00 P.M) were maintained, with ad libitum access to food and water. The rats were divided into 3 groups ( $n=8$ each). Group 1 was administered a single oral dose of $20 \mathrm{mg} / \mathrm{kg}$ TPM. Group 2 was injected a single i.p. dose of $7.5 \mathrm{mg} / \mathrm{kg}$ CP followed, seventy-two hours later, by a single oral dose of $20 \mathrm{mg} / \mathrm{kg}$ TPM. Group 3 was injected a single i.p. dose of $0.5 \mathrm{~mL} / \mathrm{kg} \mathrm{CCl}$ followed, twenty-four hours later, by a single oral dose of $20 \mathrm{mg} / \mathrm{kg}$ TPM. Biochemical parameters including Scr and AST for the three groups were measured using appropriate diagnostic kits before the pharmacokinetic study was conducted.

For the three groups, blood samples $(\sim 0.3 \mathrm{~mL}$ each $)$ were collected in preheparinized Eppendorf tubes from light etheranesthetized rats just before and at $0.25,0.5,1.0,1.5,2,4,6$, $8,12,18$, and $24 \mathrm{~h}$ following TPM administration. The blood samples were collected following the previously reported procedure [15]. The blood samples were immediately centrifuged at $9000 \times \mathrm{g}$ for $10 \mathrm{~min}$. The plasma samples were then separated and aliquots of $100 \mu \mathrm{L}$ were kept frozen at $-80^{\circ} \mathrm{C}$ pending analysis.

2.4. Plasma Samples Analysis. TPM plasma samples were analyzed using a previously described tandem mass spectrometric (LC-MS/MS) method [16]. The linear range of the method was $0.5-30 \mu \mathrm{g} / \mathrm{mL}$ and the lower limit of quantification was $0.5 \mu \mathrm{g} / \mathrm{mL}$. The intra- and interrun precisions, as measured by relative standard deviations (RSD \%), of the method were less than $8 \%$.

Prior to the assay, the frozen rat plasma samples, calibrators, and quality control samples were gently thawed at ambient temperature and then vortex-mixed for $30 \mathrm{~s}$ before extraction. To each $100 \mu \mathrm{L}$ of plasma sample, $25 \mu \mathrm{L}$ of IS $(100 \mu \mathrm{g} / \mathrm{mL})$ was added and vortex-mixed for $30 \mathrm{~s}$. To each tube, $25 \mu \mathrm{L}$ of ammonium acetate $(10 \mathrm{mM})$ and $1 \mathrm{~mL}$ of ether were added and the mixture was then centrifuged at $9000 \times \mathrm{g}$ for $10 \mathrm{~min}$. The organic layer was separated, evaporated to dryness, and then reconstituted with $100 \mu \mathrm{L}$ of the mobile phase; acetonitrile: $0.1 \%$ triethylamine $(80: 20$, v/v). A $10 \mu \mathrm{L}$ of this solution was then injected into the LC-MS/MS system.

2.5. Pharmacokinetics and Statistical Analyses. TPM pharmacokinetic parameters were estimated by standard noncompartmental methods using Kinetica software, version 5.1 (Thermo Fisher Scientific, USA). The maximum plasma concentration $\left(C_{\max }\right)$ and time needed to attain this concentration $\left(T_{\max }\right)$ were directly obtained from the drug plasma profiles; the drug plasma elimination half-life (T1/2) values were calculated as $\mathrm{Ln} 2 / \mathrm{kel}$, where kel is the elimination rate constant. The area under the plasma concentrationtime curves $\left(\mathrm{AUC}_{0-t}\right)$ was calculated from the measured data points from time zero to time of last quantifiable concentration by the linear trapezoidal rule and the area under the plasma concentration-time curves extrapolated to infinity $\left(\mathrm{AUC}_{0-\infty}\right)$ was calculated using the equation: $\mathrm{AUC}_{0-\infty}=\mathrm{AUC}_{0-t}+C^{*} / \mathrm{kel}$, where $C^{*}$ is the last quantifiable drug plasma concentration. Oral body clearance $(\mathrm{CL} / \mathrm{F})$ was calculated as $\mathrm{CL} / \mathrm{F}=$ Dose $/ \mathrm{AUC}_{0-\infty}$ and the volume of distribution $(\mathrm{Vd} / \mathrm{F})$ was calculated as $\mathrm{Vd} / \mathrm{F}=(\mathrm{CL} / \mathrm{F}) / \mathrm{kel}$. The pharmacokinetic parameters were presented as mean \pm SD. Differences between the pharmacokinetic parameters of TPM among the groups were considered statistically significant if $P<0.05$ using Mann-Whitney $U$ test (nonparametric 


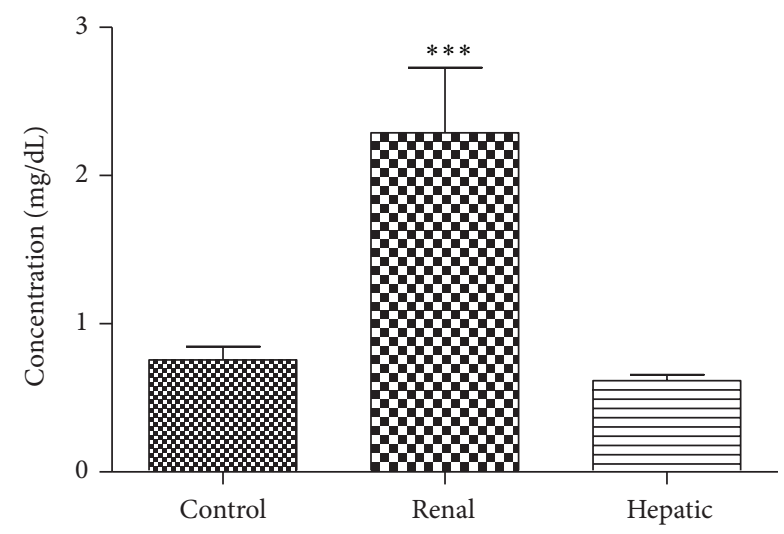

(a)

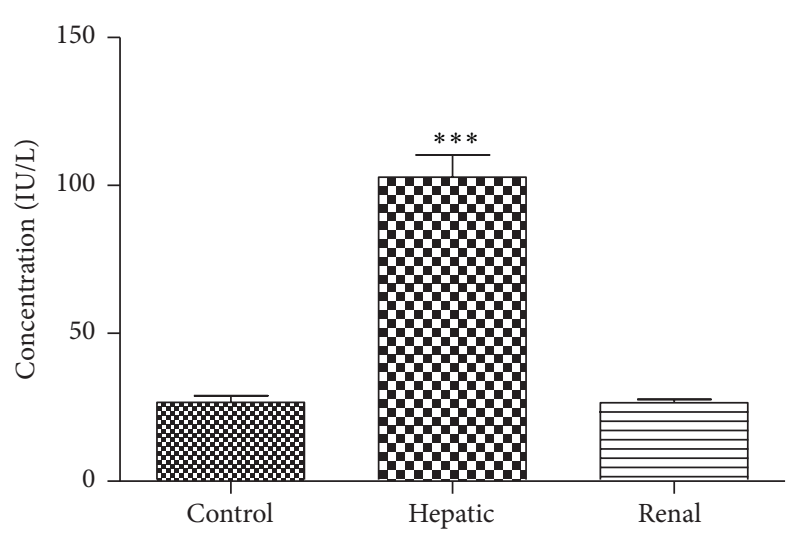

(b)

FIGURE 1: Serum creatinine (a) and aspartate transaminase (AST) (b) in control and experimentally induced hepatic or renal failure rats. Data presented are mean \pm SE in 16 rats. ${ }^{* * *} P<0.0001$, significantly different from control group.

for two independent samples). The statistical analysis was performed using the statistical package for social sciences (SPSS) software, version 20 (SPSS Inc., Chicago, IL, USA).

\section{Results}

Biochemical parameters in cisplatin-treated or $\mathrm{CCl}_{4}$-treated rats are depicted in Figure 1. Cisplatin pretreatment significantly increased serum creatinine levels (Figure 1(a)) in contrast to control rats $(P<0.0001)$. There was no difference in creatinine levels between $\mathrm{CCl}_{4}$-treated and control rats $(P>0.05)$. On the other hand, $\mathrm{CCl}_{4}$-treated rats significantly increased AST levels in comparison to control rats $(P<$ $0.0001)$, and there was no significant difference in AST levels between control and cisplatin-treated rats $(P>0.05)$, as shown in Figure 1(b).

Mean plasma concentration-time profile for TPM following an oral dose of $20 \mathrm{mg} / \mathrm{kg}$ to $\mathrm{CCl}_{4}$-treated, cisplatintreated, or control rats is demonstrated in Figure 2 and the mean pharmacokinetic parameters of TPM after an oral administration of $20 \mathrm{mg} / \mathrm{kg}$ TPM dose to the same groups are presented in Table 1. As shown in Figure 2, the plasma concentration-time profiles following an oral TPM dose $(20 \mathrm{mg} / \mathrm{kg})$ were different between $\mathrm{CCl}_{4}$-treated, cisplatintreated, and control rats. The profile in $\mathrm{CCl}_{4}$-treated and cisplatin-treated rats was decreased more slowly than that in control rats, being significantly higher in the elimination phase compared to that in control rats. However, the impact was more pronounced in $\mathrm{CCl}_{4}$-treated rats than in cisplatintreated rats. TPM oral clearance $(\mathrm{CL} / \mathrm{F})$ in $\mathrm{CCl}_{4}$-treated rats was significantly lower than that in control rats $(P<0.001)$ and was decreased by $71 \%$ in comparison to the control group, as shown in Table 1. This results in significantly higher values of $\mathrm{AUC}_{0-\infty}, \mathrm{T} 1 / 2$, and $\mathrm{Vd}$ in this group in comparison to the control group $(P<0.01)$. On the other hand, $\mathrm{CL} / \mathrm{F}$ was not significantly different between cisplatin-treated rats and control group $(P>0.05)$, as shown in Table 1 . However, the $\mathrm{T} 1 / 2$ and Vd in cisplatin-treated rats were significantly higher than in control group $(P<0.01)$.

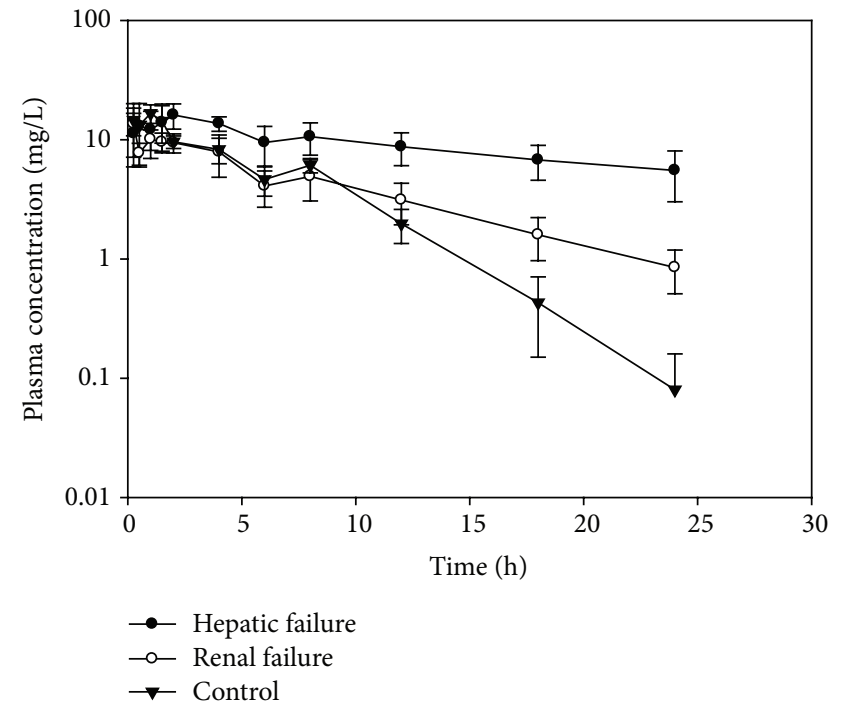

Figure 2: Plasma concentration-time profile of TPM following an oral dose of $20 \mathrm{mg} / \mathrm{kg}$ to control and experimentally induced hepatic or renal failure rats $(n=8)$.

\section{Discussion}

Patients with epilepsy may suffer from concurrent renal or hepatic diseases that can modify the pharmacokinetic profiles of antiepileptic drugs (AEDs). The present study systematically investigated the influence of compromised renal or hepatic function on TPM pharmacokinetics employing rat models of kidney or liver failure using a validated tandem mass spectrometric method (LC-MS/MS) [16]. To the best of our knowledge, this is the first study which examined the impact of these two conditions on the way body handles TPM dose. Our data demonstrated that both experimentally induced renal and hepatic failure modified the pharmacokinetic profile of this AED leading to prolongation in its $\mathrm{T} 1 / 2$. If the findings of the present study are extrapolated to humans, this may emphasize that dose adjustment of TPM 
TABLE 1: Pharmacokinetic parameters of TPM in experimentally induced hepatic or renal failure rats.

\begin{tabular}{lccc}
\hline Parameter & Control & Hepatic & Renal \\
\hline$T_{\max }(\mathrm{h})$ & $0.71 \pm 0.53$ & $1.13 \pm 0.82$ & $0.38 \pm 0.31$ \\
$C_{\max }(\mu \mathrm{g} / \mathrm{mL})$ & $17.98 \pm 2.56$ & $20.00 \pm 4.77$ & $14.43 \pm 5.77$ \\
$\mathrm{~T} 1 / 2(\mathrm{~h})$ & $2.57 \pm 0.64$ & $21.16 \pm 14.61^{\mathrm{a}}$ & $6.21 \pm 0.43^{\mathrm{b}}$ \\
$\mathrm{AUC}$ & $(\mu \mathrm{g} \cdot \mathrm{h} / \mathrm{mL})$ & $430.84 \pm 258.77^{\mathrm{a}}$ & $102.19 \pm 33.35$ \\
$\mathrm{Vd} / \mathrm{F}(\mathrm{L})$ & $93.44 \pm 17.68$ & $1.36 \pm 0.36^{\mathrm{b}}$ & $1.92 \pm 0.63^{\mathrm{b}}$ \\
$\mathrm{CL} / \mathrm{F}(\mathrm{mL} / \mathrm{h} / \mathrm{kg})$ & $0.79 \pm 0.10$ & $63.69 \pm 39.28^{\mathrm{a}}$ & $215.38 \pm 75.69$ \\
\hline
\end{tabular}

Data are shown as mean \pm SD for 8 rats.

${ }^{\text {a }}$ Significantly different $(P<0.001)$ from control.

${ }^{\mathrm{b}}$ Significantly different $(P<0.01)$ from control.

may be required when this drug is administered to epileptic patients with renal or hepatic failure. That is, therapeutic levels should be achieved in these cases by administering smaller maintenance doses or by increasing dosing intervals to avoid accumulation of this agent in the body.

Our data demonstrated that TPM $C_{\max }$ and $T_{\max }$ values were not significantly altered by experimentally induced hepatic or renal failure. This perhaps indicates that TPM's absorption was not significantly changed in both cases. However, there was a significant increase in the drug's $\mathrm{AUC}_{0-\infty}$ in $\mathrm{CCl}_{4}$-treated rats compared to cisplatin-treated or control rats. This could be due to reduced oral clearance in this group in comparison with the other two groups. In addition, the observed significant increase in $\mathrm{AUC}_{0-\infty}$ in $\mathrm{CCl}_{4}$-treated rats could be related to portal-systemic shunting, which occurs frequently in advanced liver disease. This complication may substantially decrease drug metabolism and result in a significant decrease in nonrenal clearance [17]. In accordance with our findings, Brockmöller and coworkers investigated possible changes in the pharmacokinetics of levetiracetam in patients with liver cirrhosis. Similar to TPM, this newer AED is mainly excreted unchanged in urine and is not metabolized by the liver. The investigators found that, in patients with severe cirrhosis, the drug clearance was reduced by $43 \%$ and plasma T1/2 was significantly prolonged compared with healthy subjects. The authors measured renal clearance of this AED and found that it was dramatically reduced. In addition, they reported that the decrease in renal clearance was correlated with the deterioration in renal function [9].

Based on our current findings, it is interesting to note that the mean plasma concentration-time profile of TPM, particularly in the elimination phase, decreased slowly. This observation was seen in both renal and hepatic impairment models, but it was more profound in the latter. This was accompanied by an increase in the elimination T1/2 of the drug and a significant decrease in its $\mathrm{CL} / \mathrm{F}$. It is more likely that the slower fall in TPM levels in $\mathrm{CCl}_{4}$-treated rats is related to a decrease in nonrenal clearance rather than an increase in the drug's bioavailability. TPM is predominantly excreted unchanged in urine [6], so theoretically the drug plasma concentration was expected to be higher in the renal failure group. However, the decrease in the oral clearance of the drug in $\mathrm{CCl}_{4}$-treated group could be attributed to the development of renal dysfunction in the rats which suffered from hepatic failure [18]. Paradoxically, however, this decline in kidney function did not cause rise in serum creatinine since hepatic impairment is normally accompanied by reduced serum creatinine level and resultant overestimation of creatinine clearance. Therefore, measurement of renal clearance rather than total clearance in this setting would be more useful. Compared to those who have normal renal function, TPM clearance in patients with moderate and severe renal impairment is reduced by 42 and $54 \%$, respectively [19]. Despite the fact that TPM is not extensively metabolized, it was previously demonstrated that moderateto-severe liver impairment can decrease its oral clearance by $26 \%$ [6]. In our study involving experimentally induced renal failure rats, we observed that TPM elimination T1/2 was modestly increased, whereas the $\mathrm{Vd} / \mathrm{F}$ was significantly elevated (Table 1). The significant increase in $\mathrm{Vd} / \mathrm{F}$ in this group is most likely a resultant of decreased bioavailability. However, this postulation needs further investigation. Given that TPM is normally renally excreted, the explanation for the absence of a considerable increase in the oral clearance of the drug in cisplatin-induced renal failure rats is presumably related to the significant elevation of $\mathrm{Vd} / \mathrm{F}$ which indicated that most of the drug was rather redistributed in body fluids and was not available for elimination. In accordance with our findings, Glue and coworkers evaluated the effect of renal impairment on single-dose pharmacokinetics of the newer AED, felbamate. The investigators showed that, compared to controls, total clearance of felbamate decreased while $\mathrm{T} 1 / 2$ and $\mathrm{AUC}_{0-\infty}$ values increased in subjects with renal dysfunction. The magnitude of these changes was correlated with the degree of renal dysfunction. In contrast to our findings, however, $\mathrm{Vd} / \mathrm{F}$ was lower in renal dysfunction subjects [20]. In another study, Lal and coworkers described a population-based pharmacokinetic study of gabapentin enacarbil in patients with varying degrees of renal dysfunction. The authors reported that gabapentin CL/F was proportionally related to creatinine clearance [21]. Similar to hepatic dysfunction, our data on experimentally induced renal failure indicated that dosage adjustment may be necessary in patients with compromised renal function if the present findings are extrapolated to human beings.

Based on the preceding discussion, some general observations can be made about the impact of experimentally induced liver and kidney failure on the way our body handles TPM. Overall, the findings of this study may suggest that TPM administration to patients suffering from these two 
conditions may result in prolongation in its elimination T1/2. These results emphasize the need for therapeutic drug monitoring of TPM in these settings to avoid drug accumulation and toxicity if the present findings are to be extrapolated to humans.

In conclusion, the findings of the present study suggest that the pharmacokinetic profile of TPM may be altered in the presence of compromised liver or kidney function. Further studies involving intravenous administration of TPM and including urine data are warranted to investigate the mechanisms underlying the slow fall in TPM levels in these two conditions.

\section{Abbreviations}

AST: $\quad$ Aspartate aminotransaminase

$\mathrm{CCl}_{4}$ : Carbon tetrachloride

TPM: Topiramate

T1/2: $\quad$ Elimination half-life

$C_{\max }: \quad$ Maximum plasma concentration

$T_{\max }$ : Time needed to reach maximum plasma concentration

$\mathrm{AUC}_{0-\infty}$ : Area under the plasma concentration-time curve from time zero to infinity

Vd: Apparent volume of distribution

CL/F: Oral clearance

F: $\quad$ Bioavailability factor.

\section{Conflict of Interests}

The authors declare that they have no conflict of interests.

\section{Acknowledgments}

The authors gratefully acknowledge Kuwait University Research Sector for financial support of this study (Grant no. PT02/10). The authors would like to acknowledge Animal Resources Center, HSC, Kuwait University, for providing and taking full care of the experimental animals. The technical assistance by Ms. Bindu Baby is highly appreciated.

\section{References}

[1] A. Kaminska, "New antiepileptic drugs in childhood epilepsies: indications and limits," Epileptic Disorders, vol. 3, no. 2, pp. 3746, 2001.

[2] R. Gursahani and N. Gupta, "The adolescent or adult with generalized tonic-clonic seizures," Annals of Indian Academy of Neurology, vol. 15, no. 2, pp. 81-88, 2012.

[3] Y. Liang, X. Chen, M. Osborne, S. O. DeCarlo, T. L. Jetton, and K. Demarest, "Topiramate ameliorates hyperglycaemia and improves glucose-stimulated insulin release in ZDF rats and $\mathrm{db} / \mathrm{db}$ mice," Diabetes, Obesity and Metabolism, vol. 7, no. 4, pp. 360-369, 2005.

[4] A. A. Kholin, N. N. Zavadenko, E. S. Il'Ina et al., "Efficacy and safety of topiramate depending on patient's age and forms of epilepsy," Zhurnal Nevrologii i Psihiatrii imeni S.S. Korsakova, vol. 113, no. 4, pp. 45-51, 2013.
[5] P. N. Patsalos, "Properties of antiepileptic drugs in the treatment of idiopathic generalized epilepsies," Epilepsia, vol. 46, no. 9, supplement, pp. 140-148, 2005.

[6] R. C. Sachdeo, "Topiramate: clinical profile in epilepsy," Clinical Pharmacokinetics, vol. 34, no. 5, pp. 335-346, 1998.

[7] G. W. Caldwell, W. N. Wu, J. A. Masucci et al., "Metabolism and excretion of the antiepileptic/antimigraine drug, topiramate in animals and humans," European Journal of Drug Metabolism and Pharmacokinetics, vol. 30, no. 3, pp. 151-164, 2005.

[8] V. Rodighiero, "Effects of liver disease on pharmacokinetics. An update," Clinical Pharmacokinetics, vol. 37, no. 5, pp. 399-431, 1999.

[9] J. Brockmöller, T. Thomsen, M. Wittstock, R. Coupez, H. Lochs, and I. Roots, "Pharmacokinetics of levetiracetam in patients with moderate to severe liver cirrhosis (Child-Pugh classes A, $\mathrm{B}$, and $\mathrm{C}$ ): characterization by dynamic liver function tests," Clinical Pharmacology and Therapeutics, vol. 77, no. 6, pp. 529541, 2005.

[10] R. M. Craig, P. Murphy, T. P. Gibson et al., "Kinetic analysis of D-xylose absorption in normal subjects and in patients with chronic renal failure," Journal of Laboratory and Clinical Medicine, vol. 101, no. 3, pp. 496-506, 1983.

[11] E. E. Ohnhaus, S. Vozeh, and E. Nuesch, "Absolute bioavailability of digoxin in chronic renal failure," Clinical Nephrology, vol. 11, no. 6, pp. 302-306, 1979.

[12] W. L. St. Peter, "Improving medication safety in chronic kidney disease patients on dialysis through medication reconciliation," Advances in Chronic Kidney Disease, vol. 17, no. 5, pp. 413-419, 2010.

[13] H. Wynne, "Drug metabolism and ageing," Journal of the British Menopause Society, vol. 11, no. 2, pp. 51-56, 2005.

[14] K. K. Flaharty, A. M. Grimm, and P. H. Vlasses, "Epoetin: human recombinant erythropoietin," Clinical Pharmacy, vol. 8, no. 11, pp. 769-782, 1989.

[15] K. M. Matar, "Influence of famotidine on verapamil pharmacokinetics in rats," European Journal of Drug Metabolism and Pharmacokinetics, vol. 30, no. 3, pp. 219-222, 2005.

[16] K. M. Matar, "Therapeutic drug monitoring of topiramate by liquid chromatography-tandem mass spectrometry," Clinica Chimica Acta, vol. 411, no. 9-10, pp. 729-734, 2010.

[17] M. Bosilkovska, B. Walder, M. Besson, Y. Daali, and J. Desmeules, "Analgesics in patients with hepatic impairment: pharmacology and clinical implications," Drugs, vol. 72, no. 12, pp. 1645-1669, 2012.

[18] X. Li, H. T. Hassoun, R. Santora, and H. Rabb, “Organ crosstalk: the role of the kidney," Current Opinion in Critical Care, vol. 15, no. 6, pp. 481-487, 2009.

[19] H. Stefan and T. J. Feuerstein, "Novel anticonvulsant drugs," Pharmacology and Therapeutics, vol. 113, no. 1, pp. 165-183, 2007.

[20] P. Glue, W. Sulowicz, R. Colucci et al., "Single-dose pharmacokinetics of felbamate in patients with renal dysfunction," British Journal of Clinical Pharmacology, vol. 44, no. 1, pp. 91-93, 1997.

[21] R. Lal, J. Sukbuntherng, W. Luo et al., "Clinical pharmacokinetics of gabapentin after administration of gabapentin enacarbil extended-release tablets in patients with varying degrees of renal function using data from an open-label, single-dose pharmacokinetic study," Clinical Therapeutics, vol. 34, no. 1, pp. 201-213, 2012. 

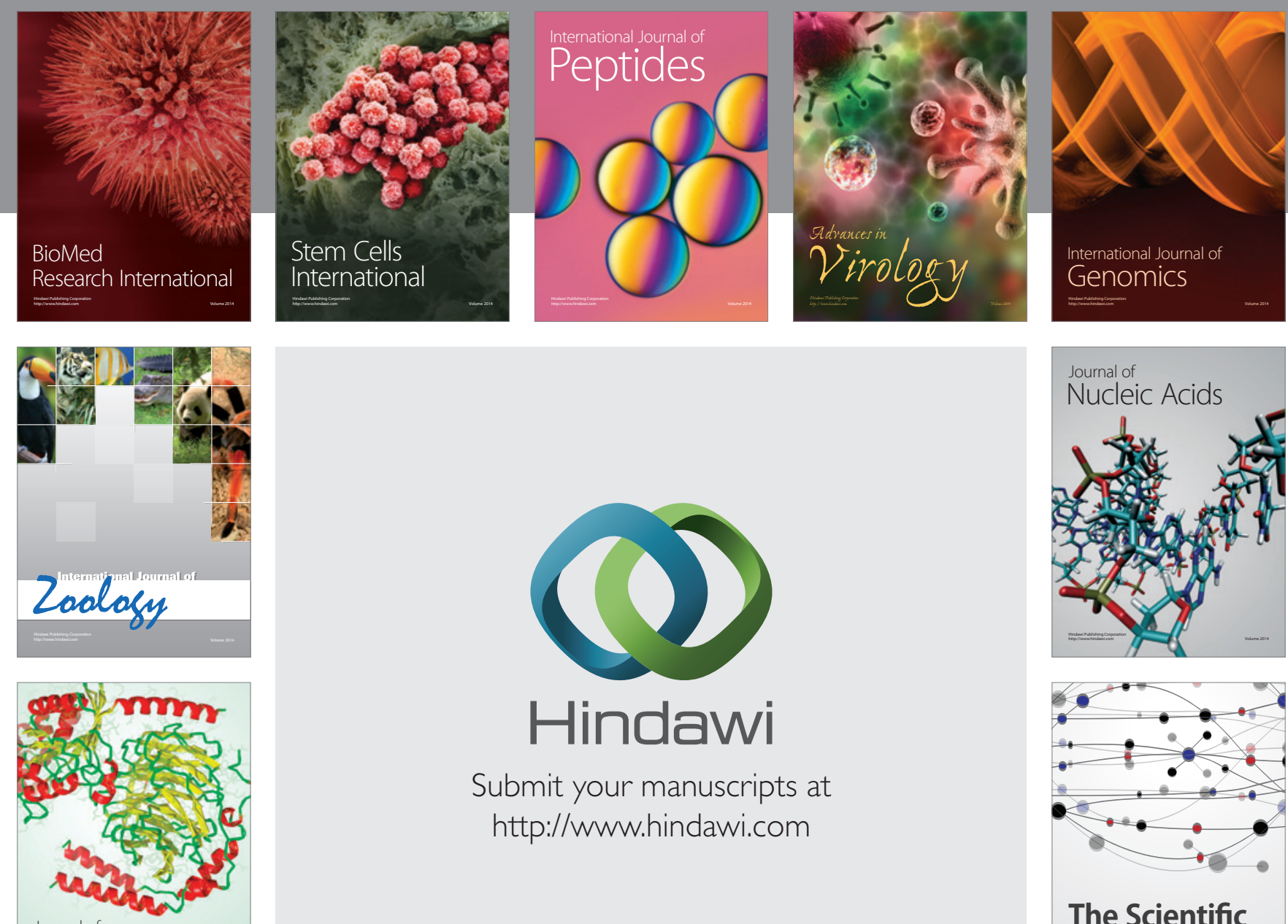

Submit your manuscripts at

http://www.hindawi.com

Journal of
Signal Transduction
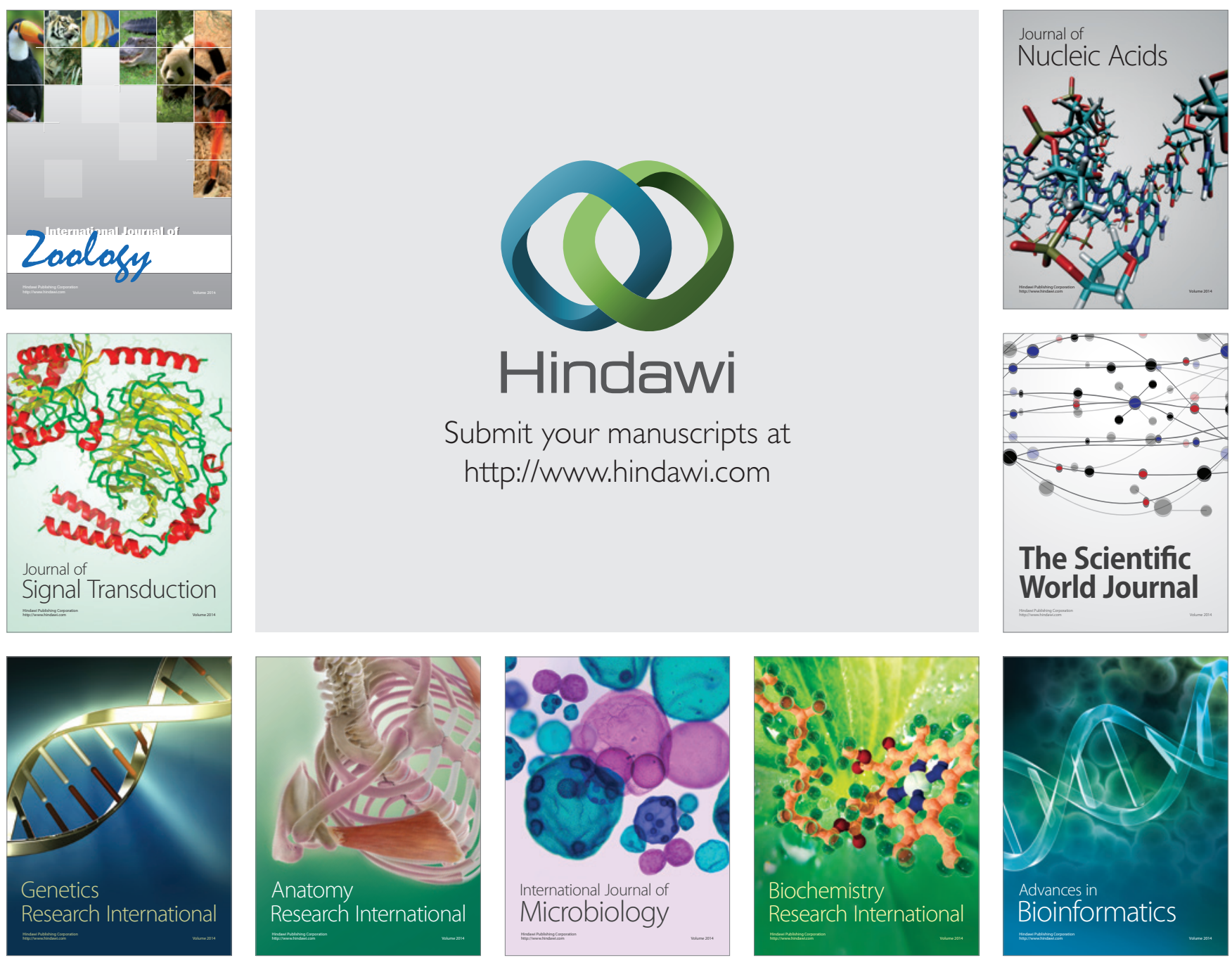

The Scientific World Journal
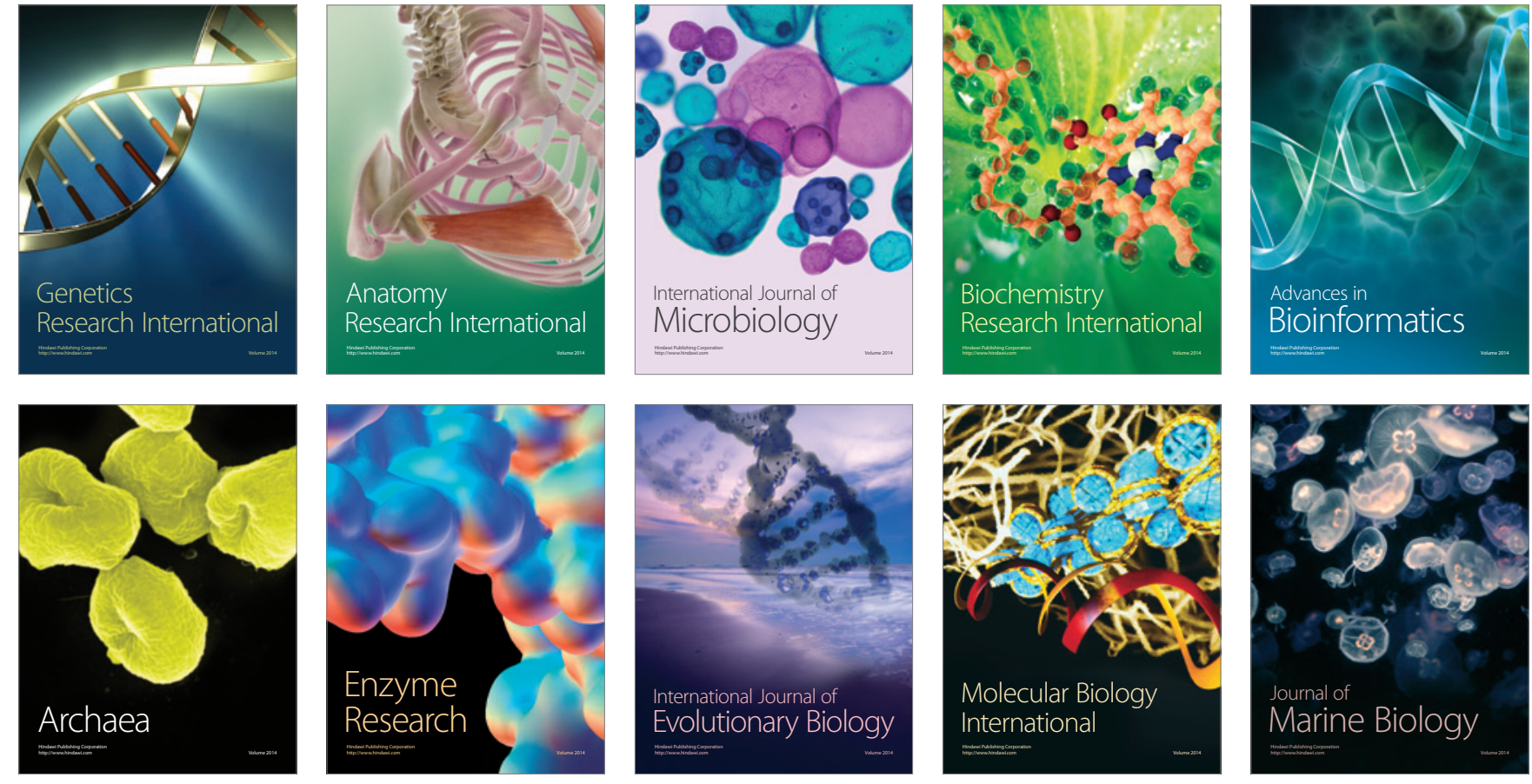